\title{
Prehospital adrenaline administration for out-of-hospital cardiac arrest: The picture in England and Wales
}

Scott Booth ${ }^{1}$, Chen Ji ${ }^{1}$, Jasmeet Soar ${ }^{2}$, A. Niroshan Siriwardena ${ }^{3}$, Rachael Fothergill ${ }^{4}$, Robert Spaight ${ }^{5}$, Gavin D. Perkins ${ }^{1,6}$, OHCAO collaborators

Purpose: Studies show significant variability in adrenaline administration between/ within EMS systems worldwide, but there is a paucity of UK data. This study aimed to describe epidemiology and outcomes for OHCA patients receiving prehospital adrenaline by EMS in the UK.

Materials and Methods: Retrospective observational study. Data on adult OHCA patients receiving adrenaline from eight English and the Welsh ambulance service regions analysed from two sources: 2010-2013 data from the PARAMEDIC RCT, and 2013-2015 data from the UK OHCA registry. Chi-square tests were used to test differences in proportions of adrenaline administration between regions. Descriptive statistics were used to report patient demographic and clinical characteristics, bystander response, EMS response time and outcomes.

Results: EMS in England and Wales treated 47,737 OHCA patients with 36,998 (77.5\%) administered adrenaline (Figure 1). Administration varied between EMS regions (Table 1, Figure 2), ranging from 62.2-86.3\% (overall difference $p<0.001$ ) for all patients, $59.9-85.3 \%$ (overall difference $p<0.001$ ) for shockable and $66.0-91.0 \%$ (overall difference $p<0.001$ ) for non-shockable rhythm patients.

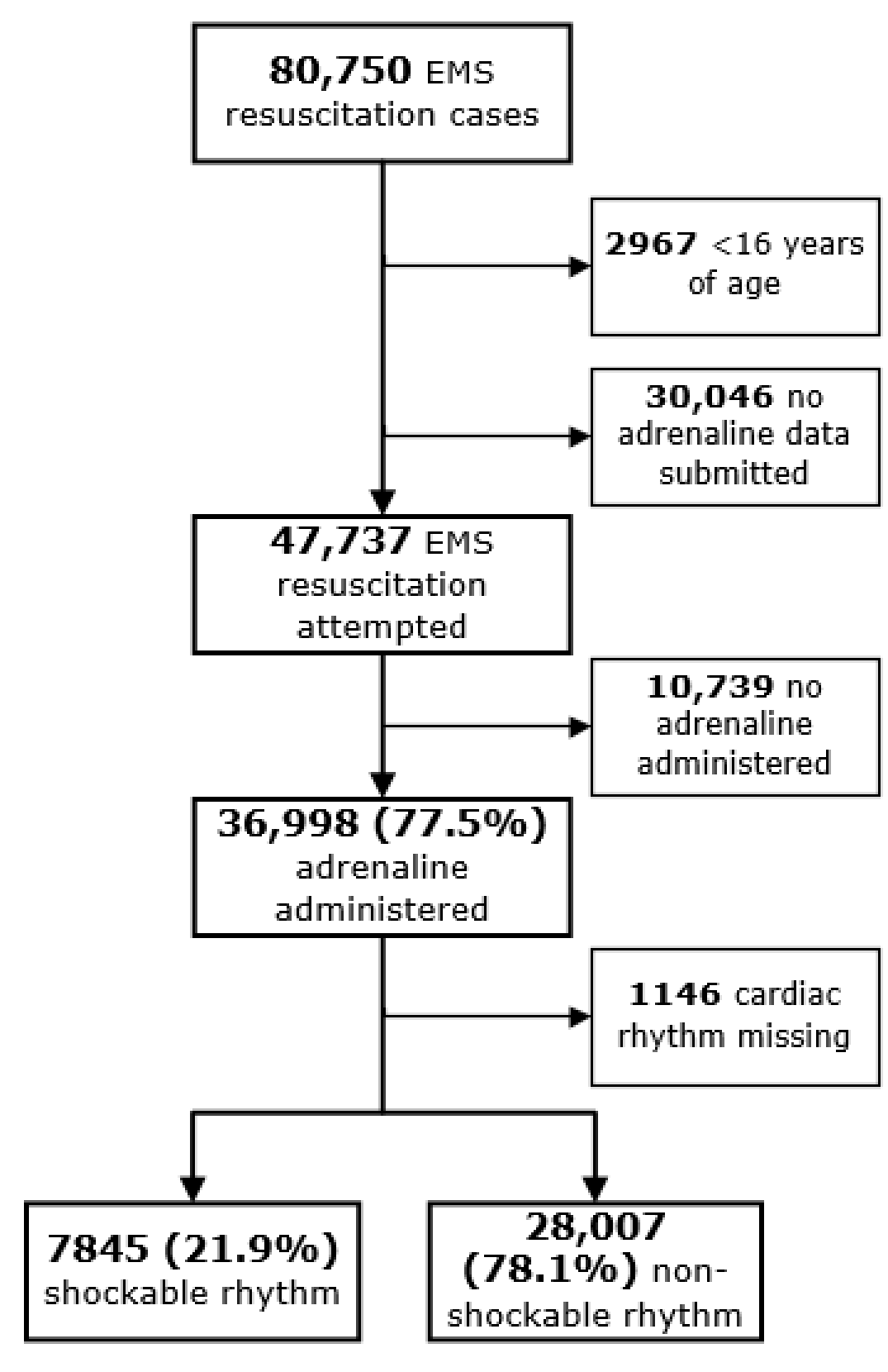

Figure 1: Data flow for OHCA patients administered adrenalin by EMS in England/Wales 2010-2015
The mean age of OHCA patients administered adrenaline by EMS was 71.0 years $(S D=16.4$ years) and $63.0 \%$ were male. The bystander CPR rate was $54.2 \%$ (for non-EMS witnessed OHCA patients). The majority of OHCA patients had a cardiac aetiology $(88.6 \%)$, whilst the initial cardiac rhythm was nonshockable in $78.1 \%$ of patients. Furthermore, the mean EMS response time was 7.8 minutes $(\mathrm{SD}=7.1$ minutes). For outcomes, the ROSC at hospital transfer rate was $22.8 \%$ and survival to discharge was $2.9 \%$ for patients administered adrenaline.
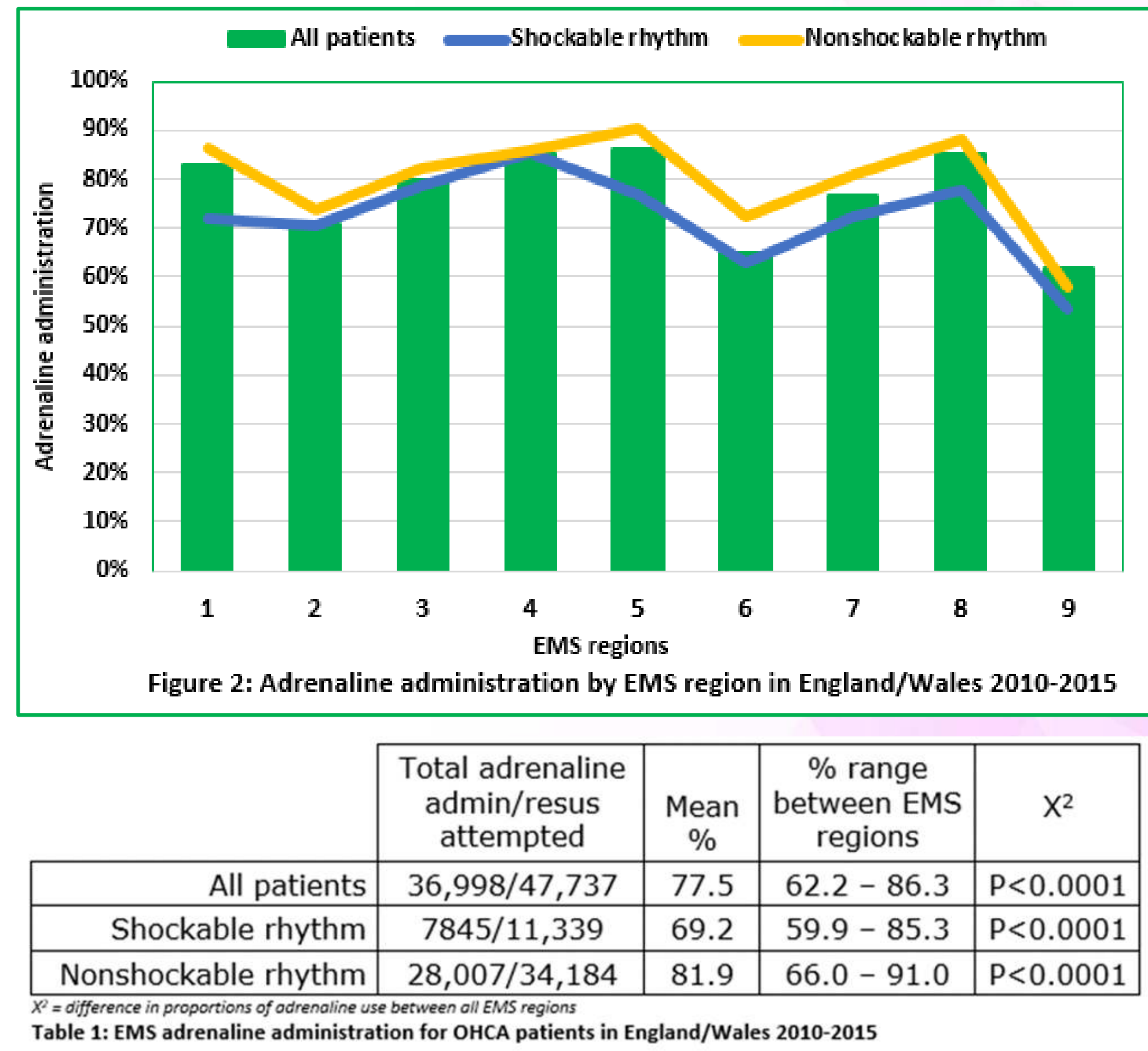

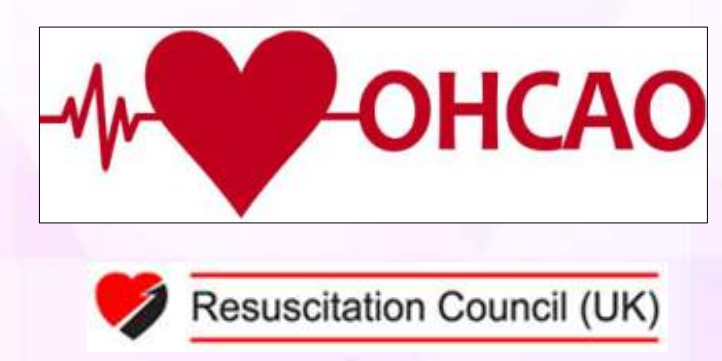

\section{(5)} Resuscitation Council (UK)

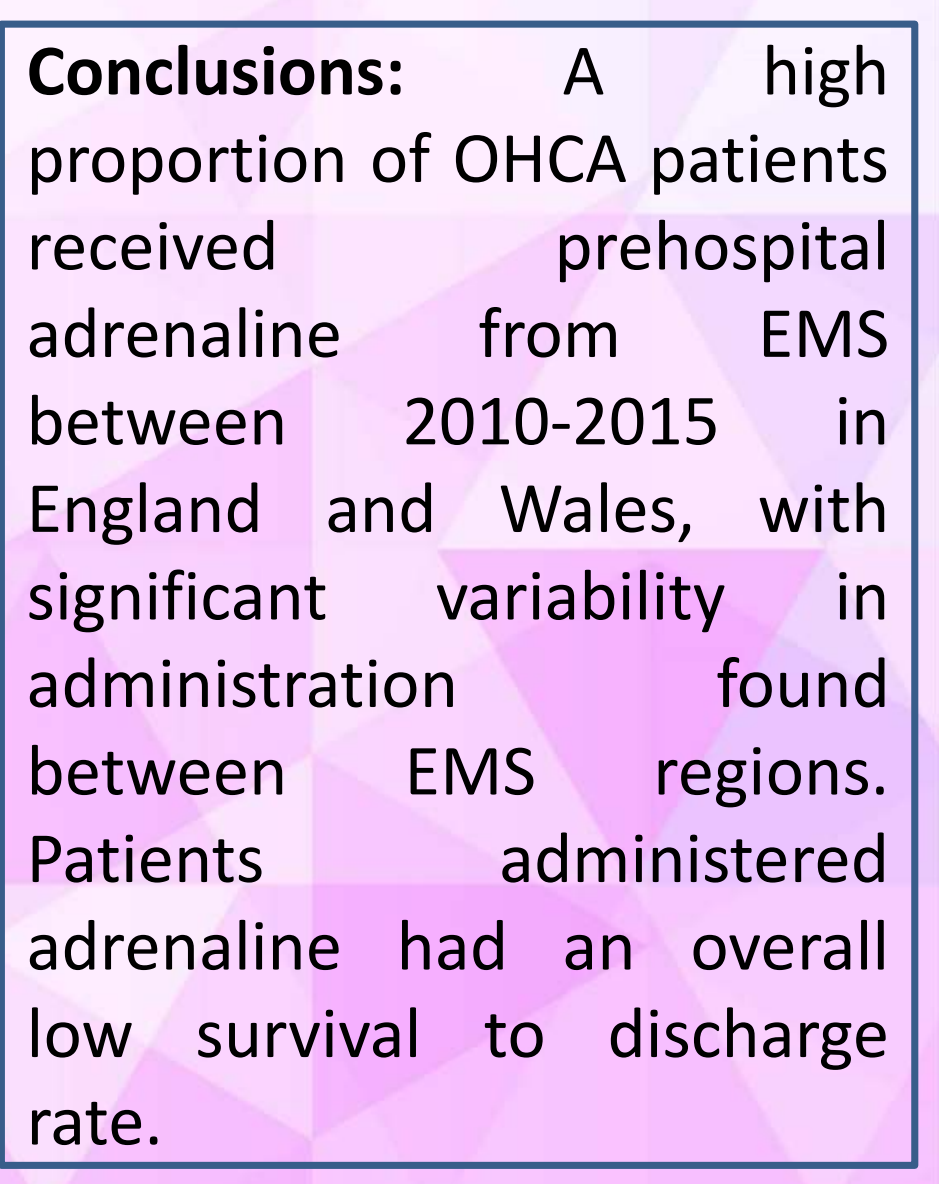
1 University of Warwick Medical School; 2 Southmead Hospital, Bristol; 3 University of Midlands Ambulance Service; 6 Heart of England NHS Foundation Trust 